\title{
Culture, gender, and the bipolarity of momentary affect
}

\author{
Michelle Yik \\ The Hong Kong University of Science and Technology, Kowloon, Hong Kong
}

\begin{abstract}
This study examined if the relation between momentary positive and negative affect varies with culture and gender. In eight samples covering five languages (English, Spanish, Chinese, Japanese, and Korean) with 3084 respondents (1305 males and 1779 females), I tested this proposal through structural equation models that controlled for random and systematic errors of measurement. In all eight samples, female respondents yielded a more negative correlation between positive and negative affect than did male respondents, but the differences were tiny and only two were statistically significant. In a multi-sample confirmatory factor analysis, the correlation was found to be substantial and negative in all five languages (Фs ranging from -.80 to -.91 ). All values from the total samples and from males and females separately were consistent with the bipolarity of positive and negative affect.
\end{abstract}

Are positive affect (PA) and negative affect (NA) polar opposites, or are they independent of, or even positively correlated with, each other? This question has vexed psychologists for half a century and its resolution touches on issues such as what strategies to use to attenuate the impact of negative affect, to enhance emotional well-being, and to cope with adversities (Diener, Smith, \& Fujita, 1995; Frederickson, 2001; Spiegel, 1998). In this paper, I focus on the bipolarity of experienced momentary affect. By momentary affect, I have in mind subjective feelings and moods that are experienced in a thin slice of time. By bipolarity, I mean that PA and NA are oppositely scored aspects of the same underlying variable (valence). Ordinarily, most people think that bipolarity predicts a correlation of -1.00 between two bipolar variables, but Russell and Carroll (1999) showed that this is not always so. Even when random and systematic errors are

Correspondence should be addressed to Michelle Yik, The Hong Kong University of Science and Technology, Division of Social Science, Clear Water Bay, Kowloon, Hong Kong. E-mail: Michelle.Yik@ust.hk

This research was supported by RGC Direct Allocation Grants (DAG02/03.HSS14 and DAG03/04.HSS14).

I thank the reviewers for their comments on the earlier drafts. I also thank Virginia Unkefer and Raymond Wong for their help in preparing this article.

(C) 2007 Psychology Press, an imprint of the Taylor \& Francis Group, an Informa business www.psypress.com/cogemotion

DOI: $10.1080 / 02699930600823702$ 
controlled, the theoretical correlation between two bipolar variables can just be as high as -.47 . The explanation for this surprising finding lies in the truncation of each variable when measured with the needed unipolar response format. For practical purposes, I argue that observed correlations between -.47 and -1.00 are highly consistent with the bipolarity of two variables.

The nature of the relation between PA and NA has long been debated. Some scholars have suggested that these two affective states are polar opposites (Green, Goldman, \& Salovey, 1993; Russell \& Carroll, 1999; Yik, Russell, \& Barrett Feldman, 1999); others argued that they are independent (Bradburn, 1969; Watson \& Tellegen, 1985). Still others provided evidence demonstrating both bipolarity and independence in their data depending on the time frame, the level of analysis, and the typicality of the occasion (Diener \& Emmons, 1984; J. T. Larsen, McGraw, \& Cacioppo, 2001; Scollon, Diener, Oishi, \& Biswas-Diener, 2005; Zautra, Potter, \& Reich, 1997).

To complicate the debate further, several authors have recently marshalled evidence showing that culture is an important moderator of the relation between PA and NA (e.g., Bagozzi, Wong, \& Yi, 1999; Schimmack, Oishi, \& Diener, 2002; Scollon et al., 2005). They argued that PA and NA are not intrinsically related. Rather, their relation is dictated by the dominant cultural tradition of the person experiencing the emotion. People from different cultural traditions think about and therefore experience emotion differently. In particular, people from the Aristotelian Western cultural tradition tend to experience PA and NA as opposites; whereas those from the Confucian Eastern cultural tradition tend to experience them as compatible. Western thought, with its independent construal of self, leads to the experience of emotion in an oppositional or polar way; Eastern thought, with its interdependent construal of self, leads to the experience of emotion in a dialectic or harmonious way (Markus \& Kitayama, 1991; Peng \& Nisbett, 1999; Spencer-Rodgers, Peng, Wang, \& Hou, 2004). Moreover, within each cultural tradition, females, compared with males, are more emotionally knowledgeable and more subject to the ideology of emotion embedded in their cultural heritage (Brody \& Hall, 1993; Lutz, 1996).

In a tricultural study, Bagozzi et al. (1999) offered correlational evidence from American, PRC Chinese, and Korean respondents in support of the interaction effect between culture and gender on the experience of emotion. In their American sample, they found a strong negative correlation between PA and NA among female respondents but a weaker negative correlation among male respondents. In their Chinese sample, in contrast, they found a strong positive correlation among female respondents but a weak positive correlation among male respondents. Results from the Korean sample were similar to those from the Chinese sample. In another cross-cultural examination of the bipolarity thesis, Schimmack et al. (2002) found strong 
support for cultural differences in the PA-NA correlation but weak support for gender differences in their multi-level models (see also Scollon et al., 2005). Similarly, Kitayama, Markus, and Kurokaway (2000) reported cultural differences in comparing their American and Japanese samples.

Although these studies demonstrated important support for the cultural variation in the relation between PA and NA, there are several reasons why they do not provide a convincing test of the bipolarity thesis. First, most of the evidence for the dialectical model of emotions was based on reports over an extended time frame, including intensity "in general" and frequency in the "past month" (Kitayama et al., 2000; Schimmack et al., 2002), with the exception of the studies by Bagozzi et al. (1999) and Scollon et al. (2005). Bipolarity does not rule out feeling positive at one time and negative at another over an extended period of time. It simply pinpoints the fact that in one thin slice of time, one cannot feel both positive and negative.

Even a questionnaire with the "right now" instruction (such as the ratings completed by respondents in Bagozzi et al.'s [1999] study) can be problematic because its instructions are conceptually ambiguous: When respondents are asked about feeling "guilty" or "love", they seem to be invited to construe the "right now" as something like "this period in my life". Bipolarity does not rule out loving someone during the same period as feeling guilty. Indeed, in some circumstances, love is manifested as feeling guilty. Moreover, respondents' "right now" feelings might change from item to item over the course of completing a questionnaire and the resulting ratings might indeed represent the affect of an aggregate of several moments.

Scollon et al. (2005) examined the bipolarity thesis in five cultures by requesting that participants provide seven reports of affect every day for seven consecutive days. The resulting data provided a platform on which the structure of both momentary and trait affect could be examined. In terms of momentary affect, the correlations between PA and NA in five samples were substantial and negative. In terms of trait affect (i.e., the frequency of experiencing an emotion), however, PA and NA were positively correlated in Asian samples but uncorrelated in non-Asian samples. A dialectical model of emotion was used to explain the results. Asian participants were said to be socialised to be capable of experiencing both positive and negative emotions in a harmonious way (Peng \& Nisbett, 1999). To compute their frequency data, Scollon et al. summed the number of occasions on which an emotion was reported by participants. In other words, the data were aggregated over different occasions of seven days. I contend that bipolarity does not preclude feeling happy on one occasion and sad on another over the course of seven days. Perhaps, Asians are sensitive to and hence are willing to report all sorts of emotions, be they positive or negative, over an extended period of time.

Other previous studies defined bipolarity as the correlation between two composite scores that they called PA and NA. As Russell and Carroll (1999) 
stated, "Bipolarity has not been challenged at the level of such specific items as happy and sad or guilty and innocent but rather at a more abstract level: factors or scales named positive and negative" (p. 6). Each composite score was composed of a diverse collection of terms that vary along the pleasuredispleasure dimension as well as other dimensions. For instance, Kitayama et al. (2000) found that in the Japanese sample, the interpersonally disengaged positive emotion cluster was positively related to the interpersonally engaged negative emotion cluster. The resulting correlation was used as a piece of evidence supporting the dialectical model of emotion. However, a careful examination of the two clusters revealed that the former cluster consists of "proud, superior, top of the world" and the latter "ashamed, guilty, indebted". The PA and NA were positively related possibly because both were perceived to be socially undesirable in East Asian countries where feeling proud and superior is as negative as feeling ashamed.

Moreover, the response format is another nagging issue. With response options anchored by "very slightly or not at all" at one end and "very much" at the other, this "ambiguous-likely unipolar" format is open to various interpretations (see Russell \& Carroll, 1999). If so, it is possible that the cultural differences obtained in previous studies stem not from differences in emotional experience but from different interpretations of the response format. Specifically, it is conceivable that the American respondents construed the option "very slightly or not at all" as restricted to the item's polar opposite, but that the Chinese respondents construed that same response option as including a neutral feeling. The difference in the perception of the options could render a more negative correlation between positive and negative affect for Americans than for Chinese. Indeed, a dialectical model of the world view of these cultures is consistent with this more cognitive interpretation of their data.

\section{The current study}

To test bipolarity, the affect that is supposed to be bipolar to another affect must be specified (Cacioppo \& Bernson, 1994; Russell \& Carroll, 1999). In the current study, I used an explicit model of affect to guide my item selection in testing bipolarity. I relied on a two-dimensional affective space defined by the bipolar axes of Pleasure versus Displeasure and Activation versus Deactivation. The space has been shown to be capable of integrating the major dimensional models of affect including Russell's (1980) circumplex, Thayer's (1996) energetic and tense arousal, R. J. Larsen and Diener's (1992) eight combinations of pleasantness and activation, and Watson and Tellegen's (1985) positive and negative affect. The integrated space has demonstrated cross-language generalisability with replications in English, 
Spanish, Chinese, Japanese, and Korean (Yik \& Russell, 2001, 2003; Yik, Russell, \& Ahn, 2003a; Yik, Russell, Oceja, \& Fernández Dols, 2000; Yik, Russell, \& Suzuki, 2003b). A model of the entire affective space allowed me to delineate and isolate more precisely the axis of positive versus negative valence (Yik, Russell, \& Feldman Barrett, 1999; Yik, Russell, \& Steiger, 2007). Specifically, I used two separate scales of Pleasure and Displeasure to capture the anchoring points of the horizontal axis of that affective space. They were found to be approximately 180 degrees apart within the twodimensional model in the above languages. Hence, these two unipolar scales allowed me to test the degree of bipolarity in eight different language samples.

To isolate a single moment in time, I used a "remembered moments" method (Yik, Russell, Ahn, Fernández Dols, \& Suzuki, 2002). I believe that this method has advantages over the more typical method such as the "right now" instructions used in previous studies. I asked respondents to recall and then think about a single moment from the previous day. The resulting sample of moments from the respondents is thus likely to be more representative of experiences in the external, non-laboratory world. Each item on the questionnaire was to be answered with respect to this predefined moment. This method's downside is its reliance on memory. To minimise this problem, respondents were asked to select a specific moment that was well remembered, and mealtimes were used as mnemonic anchors. At the same time, remembered moments allow respondents to rely on culturally influenced emotion schemas. Thus, I expect the "remembered moments" method to provide the best testing ground for the dialectical model of emotion.

For the past several years, the bipolarity debate has been complicated by the presence of measurement errors. Recently, Green et al. (1993) and Feldman Barrett and Russell (1998) showed that random and systematic errors inherent in measurement can obscure the underlying structure: Data gathered with different response formats and then analysed with a structural equation model revealed simple and powerful relations not evident in observed measures (see also Diener et al., 1995). While agreeing with Bagozzi et al.'s (1999, pp. 642-643) observation that "the evidence for bipolarity is virtually the same between random error and systematic error models", I note that systematic error models improve the fit indices of the tested models. Indeed, Green et al. argued that the use of different response formats in gathering the data results in a correlation matrix that has already reduced the influence of systematic errors even in random error models. To control for random and systematic errors in the present study, I assessed each affect construct with three scales, each in a different response format.

Russell and Carroll (1999) noted a contradiction in previous analyses of bipolarity that had required unipolar response formats and a correlation 
of -1 . These two requirements cannot be met simultaneously. Even when random and systematic errors have been completely eliminated, to achieve a correlation of -1 requires a strictly bipolar response format. When unipolar formats are used, the more strictly unipolar the format is, the farther from -1 is the expected correlation between polar opposites. With the "ambiguous-likely unipolar" formats, such as the Adjective format used in the current study, one cannot simply calculate a correlation and require that it be close to -1 . Rather, bipolarity predicts correlations within the range between -.47 and -1.00 . The closer the correlation is to -1 , of course, the more confident one is that there is bipolarity. By "correlation", here, I refer to the correlation estimated by a structural equation modelling procedure that takes into account both random and systematic errors.

The current study extends previous efforts to move beyond the English language boundary: I examine bipolarity in eight samples covering five different languages (English, Spanish, Chinese, Japanese, and Korean), from four distinct language families. Language families are groups of languages that share a common historical origin and have similar grammar and syntax (Crystal, 1997). Spanish, like English, belongs to the Indo-European language family, although the former is under the Italic branch and the latter under the Germanic branch. Chinese belongs to the Sino-Tibetan family. Although many Westerners might assume that Chinese, Japanese, and Korean are similar languages, they actually represent different language families. Japanese is a language family of its own and is very different from any other language. Korean is a member of the Altaic family (which includes Turkish and Mongolian).

\section{METHOD}

\section{Respondents}

The present study relied on reanalyses of eight samples covering five languages (3 English, 2 Chinese, 1 each from Spanish, Japanese, and Korean) the data from which were analysed for the structure of affect and the results reported elsewhere (Yik \& Russell, 2001, 2003; Yik et al., 1999, 2000, 2003a, 2003b, 2007). All respondents were undergraduate students who took part in the study as volunteers. Test administration took place either during class time or in a laboratory.

The English sample sizes were 217 (English 1; mean age =21.3), 535 (English 2; mean age $=19.6$ ), and 395 (English 3; mean age $=19.6$ ). The Chinese sample sizes were 487 (Chinese 1 ; mean age $=19.9$ ), and 402 (Chinese 2; mean age $=20.2$ ). The Spanish sample size was 233 (mean age $=$ 19.8). The Japanese sample size was 450 (mean age $=19.7$ ). The Korean sample size was 365 (mean age $=21.2$ ). 


\section{Affect questionnaires}

Instructions. I adopted the "Remembered Moments Questionnaire" technique in all but English Sample 1. (For English Sample 1, respondents were asked to report their current mood.) Respondents were asked to recall a specific moment from the previous day with a particular anchoring time. Six anchoring times were used: "before breakfast," "after breakfast," "before lunch," "after lunch," "before dinner," and "after dinner."1 Respondents were randomly assigned to one of the prescribed times and given instructions. For instance, the instructions for the "after breakfast" version were as follows:
... we need to ask you to remember a particular moment. Please think back to yesterday. Specifically, recall the time just after breakfast. (If you didn't have breakfast yesterday, simply recall that approximate time of day.)
It is important that you remember a specific moment accurately. So, please search your memory and try to recall where you were, what you were doing at that time, who you were with, and what you were thinking.
Now select a particular moment that is especially clear in your memory. (If you really have no recollection of the time just after breakfast, please search your memory for the closest time that you do recall accurately.)

In the other five versions, the italicised words were replaced with other anchoring times. The instructions then emphasised that all subsequent questionnaires were to be answered with respect to that selected moment from the day before.

Response formats. Respondents received a battery of three affect questionnaires, each in a different response format, in the following order: (1) the Adjective format, abbreviated ADJ, which was a list of adjectives accompanied by a 5-point Likert scale ranging from 1 "not at all" to 5 "extremely"; (2) the "Agree-Disagree" format, abbreviated AGR, which was a list of statements on each of which respondents were asked to indicate their degree of agreement, ranging from 1 "strongly disagree" to 5 "strongly agree"; and (3) the "Describes-Me" format, abbreviated DES, which was a list of statements on each of which respondents were asked to indicate how well the statement described their feelings, ranging from 1 "not at all" to 4 "very well".

For each response format, the Pleasure scale (happy, satisfied, pleased, content) and the Displeasure scale (unhappy, dissatisfied, miserable, troubled) were each computed as an average of the format's constituent items. ${ }^{2}$

\footnotetext{
${ }^{1}$ In Chinese Sample 2, only "after breakfast", "after lunch", and "after dinner" were used.

${ }^{2}$ In English Sample 2, satisfied and dissatisfied were not included.
} 
Respondents in all eight samples completed items on affect in all three questionnaires. They also completed other scales not pertinent to the present study.

Translations. All instructions and scales had been translated into Spanish, Chinese, Japanese, and Korean from English by two bilingual translators, in each language. A back-translation procedure was adopted (Brislin, 1970). For instance, one bilingual translator translated the English version into Spanish. Another bilingual translator, who was blind to the English original, translated the Spanish version back into English. Discrepancies between the English and Spanish versions were then reviewed by the authors and the bilingual translators. The translations were revised until the discrepancies were resolved.

\section{RESULTS}

\section{Zero-order correlations}

I begin with correlations between individual items from the hypothesised polar opposite scales, Pleasure and Displeasure. The fourth to sixth columns of Table 1 provide descriptive statistics for the inter-item correlations between the constituent items of Pleasure and Displeasure. For each sample, results are reported for the total sample and for male and female subsamples. Let me illustrate using the total sample of English Sample 1. For the Adjective format, correlations were computed between all possible pairs of items, one from the Pleasure scale and one from the Displeasure scale; correlations ranged from -.33 to -.54 (mean $=-.43 ; S D=0.06$ ). All of the 72 mean inter-item correlations presented in Table 1 are negative; the coefficients range from -.40 to -.74 .

The seventh column of Table 1 provides the alpha coefficients for the Pleasure and Displeasure scales. With the exception of the "Describes-Me" Pleasure scale for the Japanese respondents, the coefficients for Pleasure ranged from .78 to .94 and those for Displeasure ranged from .78 to .93 , indicating that the scales were internally consistent. The last column of Table 1 gives the correlation between the Pleasure and Displeasure scale scores within each response format. Values ranged from -.51 to -.86 , lending initial support to bipolarity. Consistent with the results reported by Russell and Carroll (1999), the correlations here varied as a function of the response format. The Adjective format (ambiguous-likely unipolar) yielded weaker negative correlations than did the Agree-Disagree and DescribesMe formats (ambiguous-likely bipolar). 
TABLE 1

Pleasure (P) versus Displeasure (D): Inter-item and inter-scale zero-order correlations

\begin{tabular}{|c|c|c|c|c|c|c|c|}
\hline \multirow[b]{2}{*}{ Language } & \multirow[b]{2}{*}{ Sample } & \multirow{2}{*}{$\begin{array}{l}\text { Response } \\
\text { format }\end{array}$} & \multicolumn{3}{|c|}{ Inter-item correlations } & \multirow[b]{2}{*}{$\alpha_{P} / \alpha_{D}$} & \multirow{2}{*}{$\begin{array}{c}P-D \\
\text { correlation }\end{array}$} \\
\hline & & & Range & Mean & $S D$ & & \\
\hline \multirow[t]{9}{*}{ English 1} & Total & ADJ & -.33 to -.54 & -.43 & .06 & $.83 / .86$ & -.55 \\
\hline & & AGR & -.58 to -.72 & -.64 & .05 & $.89 / .88$ & -.79 \\
\hline & & DES & -.54 to -.71 & -.61 & .06 & $.78 / .89$ & -.75 \\
\hline & Males & ADJ & -.29 to -.52 & -.40 & .10 & $.87 / .84$ & -.51 \\
\hline & & AGR & -.54 to -.76 & -.64 & .08 & $.89 / .86$ & -.80 \\
\hline & & DES & -.44 to -.67 & -.55 & .08 & $.81 / .87$ & -.68 \\
\hline & Females & ADJ & -.34 to -.54 & -.43 & .06 & $.81 / .87$ & -.57 \\
\hline & & AGR & -.57 to -.71 & -.64 & .05 & $.89 / .89$ & -.78 \\
\hline & & DES & -.57 to -.73 & -.63 & .06 & $.78 / .90$ & -.77 \\
\hline \multirow[t]{9}{*}{ English 2} & Total & ADJ & -.48 to -.61 & -.55 & .04 & $.91 / .89$ & -.71 \\
\hline & & AGR & -.64 to -.76 & -.70 & .04 & $.92 / .89$ & -.83 \\
\hline & & DES & -.66 to -.77 & -.71 & .04 & $.86 / .90$ & -.83 \\
\hline & Males & ADJ & -.36 to -.59 & -.48 & .06 & $.88 / .89$ & -.65 \\
\hline & & AGR & -.54 to -.73 & -.65 & .06 & $.92 / .89$ & -.81 \\
\hline & & DES & -.58 to -.73 & -.65 & .06 & $.86 / .90$ & -.80 \\
\hline & Females & ADJ & -.55 to -.66 & -.60 & .03 & $.93 / .89$ & -.75 \\
\hline & & AGR & -.69 to -.78 & -.73 & .03 & $.92 / .89$ & -.85 \\
\hline & & DES & -.70 to -.80 & -.74 & .04 & $.86 / .90$ & -.85 \\
\hline \multirow[t]{9}{*}{ English 3} & Total & ADJ & -.47 to -.65 & -.57 & .06 & $.91 / .89$ & -.74 \\
\hline & & AGR & -.48 to -.76 & -.66 & .11 & $.91 / .90$ & -.83 \\
\hline & & DES & -.61 to -.79 & -.71 & .06 & $.90 / .93$ & -.86 \\
\hline & Males & ADJ & -.41 to -.68 & -.57 & .07 & $.90 / .89$ & -.75 \\
\hline & & AGR & -.44 to -.76 & -.66 & .10 & $.91 / .90$ & -.83 \\
\hline & & DES & -.63 to -.84 & -.72 & .06 & $.90 / .93$ & -.86 \\
\hline & Females & ADJ & -.43 to -.68 & -.58 & .07 & $.91 / .89$ & -.74 \\
\hline & & AGR & -.46 to -.76 & -.66 & .11 & $.91 / .90$ & -.83 \\
\hline & & DES & -.57 to -.78 & -.70 & .07 & $.90 / .93$ & -.85 \\
\hline \multirow[t]{9}{*}{ Chinese 1} & Total & ADJ & -.39 to -.62 & -.52 & .07 & $.91 / .90$ & -.68 \\
\hline & & AGR & -.52 to -.68 & -.61 & .05 & $.90 / .86$ & -.76 \\
\hline & & DES & -.65 to -.67 & -.66 & .01 & $.89 / .90$ & -.76 \\
\hline & Males & ADJ & -.41 to -.64 & -.51 & .07 & $.91 / .90$ & -.66 \\
\hline & & AGR & -.50 to -.66 & -.57 & .05 & $.90 / .86$ & -.71 \\
\hline & & DES & -.58 to -.67 & -.62 & .04 & $.89 / .90$ & -.72 \\
\hline & Females & ADJ & -.36 to -.61 & -.53 & .08 & $.91 / .90$ & -.69 \\
\hline & & AGR & -.54 to -.72 & -.63 & .06 & $.89 / .86$ & -.79 \\
\hline & & DES & -.66 to -.70 & -.68 & .02 & $.89 / .90$ & -.78 \\
\hline \multirow[t]{3}{*}{ Chinese 2} & Total & $\mathrm{ADJ}$ & -.32 to -.55 & -.43 & .07 & $.87 / .87$ & -.60 \\
\hline & & AGR & -.45 to -.60 & -.54 & .07 & $.85 / .84$ & -.71 \\
\hline & & DES & -.51 to -.66 & -.61 & .06 & $.85 / .86$ & -.74 \\
\hline
\end{tabular}


Table 1 (Continued)

\begin{tabular}{|c|c|c|c|c|c|c|c|}
\hline \multirow[b]{2}{*}{ Language } & \multirow[b]{2}{*}{ Sample } & \multirow{2}{*}{$\begin{array}{l}\text { Response } \\
\text { format }\end{array}$} & \multicolumn{3}{|c|}{ Inter-item correlations } & \multirow[b]{2}{*}{$\alpha_{P} / \alpha_{D}$} & \multirow[b]{2}{*}{$\begin{array}{c}P-D \\
\text { correlation }\end{array}$} \\
\hline & & & Range & Mean & $S D$ & & \\
\hline & Males & $\mathrm{ADJ}$ & -.25 to -.54 & -.40 & .08 & $.86 / .87$ & -.57 \\
\hline & & AGR & -.41 to -.61 & -.51 & .07 & $.85 / .84$ & -.69 \\
\hline & & DES & -.47 to -.64 & -.57 & .06 & $.85 / .86$ & -.70 \\
\hline & Females & ADJ & -.29 to -.55 & -.46 & .07 & $.89 / .87$ & -.62 \\
\hline & & AGR & -.49 to -.65 & -.57 & .07 & $.85 / .84$ & -.71 \\
\hline & & DES & -.59 to -.71 & -.66 & .05 & $.85 / .86$ & -.79 \\
\hline \multirow[t]{9}{*}{ Spanish } & Total & ADJ & -.36 to -.72 & -.56 & .13 & $.93 / .85$ & -.75 \\
\hline & & AGR & -.47 to -.74 & -.65 & .10 & $.93 / .86$ & -.79 \\
\hline & & DES & -.58 to -.75 & -.64 & .07 & $.87 / .83$ & -.79 \\
\hline & Males & ADJ & -.32 to -.70 & -.52 & .12 & $.94 / .85$ & -.69 \\
\hline & & AGR & -.49 to -.76 & -.64 & .09 & $.93 / .86$ & -.75 \\
\hline & & DES & -.49 to -.71 & -.62 & .08 & $.89 / .83$ & -.73 \\
\hline & Females & ADJ & -.35 to -.74 & -.59 & .14 & $.93 / .85$ & -.80 \\
\hline & & AGR & -.46 to -.75 & -.65 & .10 & $.93 / .86$ & -.82 \\
\hline & & DES & -.57 to -.77 & -.66 & .09 & $.89 / .83$ & -.83 \\
\hline \multirow[t]{9}{*}{ Japanese } & Total & $\mathrm{ADJ}$ & -.25 to -.54 & -.43 & .10 & $.93 / .86$ & -.57 \\
\hline & & AGR & -.43 to -.67 & -.57 & .09 & $.94 / .85$ & -.69 \\
\hline & & DES & -.20 to -.55 & -.40 & .15 & $.62 / .80$ & -.56 \\
\hline & Males & $\mathrm{ADJ}$ & -.24 to -.55 & -.41 & .10 & $.94 / .86$ & -.53 \\
\hline & & AGR & -.36 to -.66 & -.52 & .10 & $.94 / .85$ & -.64 \\
\hline & & DES & -.22 to -.50 & -.36 & .13 & $.62 / .80$ & -.51 \\
\hline & Females & ADJ & -.25 to -.53 & -.45 & .10 & $.93 / .86$ & -.61 \\
\hline & & AGR & -.50 to -.72 & -.63 & .09 & $.94 / .85$ & -.76 \\
\hline & & DES & -.18 to -.62 & -.44 & .18 & $.62 / .80$ & -.61 \\
\hline \multirow[t]{9}{*}{ Korean } & Total & $\mathrm{ADJ}$ & -.35 to -.60 & -.47 & .08 & $.93 / .86$ & -.61 \\
\hline & & AGR & -.61 to -.71 & -.68 & .03 & $.92 / .89$ & -.80 \\
\hline & & DES & -.39 to -.69 & -.56 & .14 & $.83 / .78$ & -.74 \\
\hline & Males & $\mathrm{ADJ}$ & -.35 to -.60 & -.47 & .07 & $.93 / .86$ & -.61 \\
\hline & & AGR & -.60 to -.69 & -.66 & .03 & $.92 / .89$ & -.78 \\
\hline & & DES & -.36 to -.66 & -.53 & .13 & $.83 / .78$ & -.69 \\
\hline & Females & $\mathrm{ADJ}$ & -.30 to -.62 & -.45 & .09 & $.92 / .86$ & -.62 \\
\hline & & AGR & -.62 to -.74 & -.69 & .04 & $.92 / .89$ & -.82 \\
\hline & & DES & -.37 to -.73 & -.59 & .16 & $.83 / .78$ & -.78 \\
\hline
\end{tabular}

Note: $N$ for English $1=217$ (56 males and 161 females); $N$ for English $2=535$ (241 males and 294 females); $N$ for English $3=395$ (144 males and 251 females); $N$ for Chinese $1=487$ (164 males and 323 females); $N$ for Chinese $2=402$ (200 males and 202 females); $N$ for Spanish $=233$ ( 96 males and 137 females); $N$ for Japanese $=450$ (228 males and 222 females); $N$ for Korean $=365$ (176 males and 189 females).

$\mathrm{ADJ}=$ "Adjective" format; AGR = "Agree-Disagree" format; DES = "Describes-Me" format. 


\section{YIK}

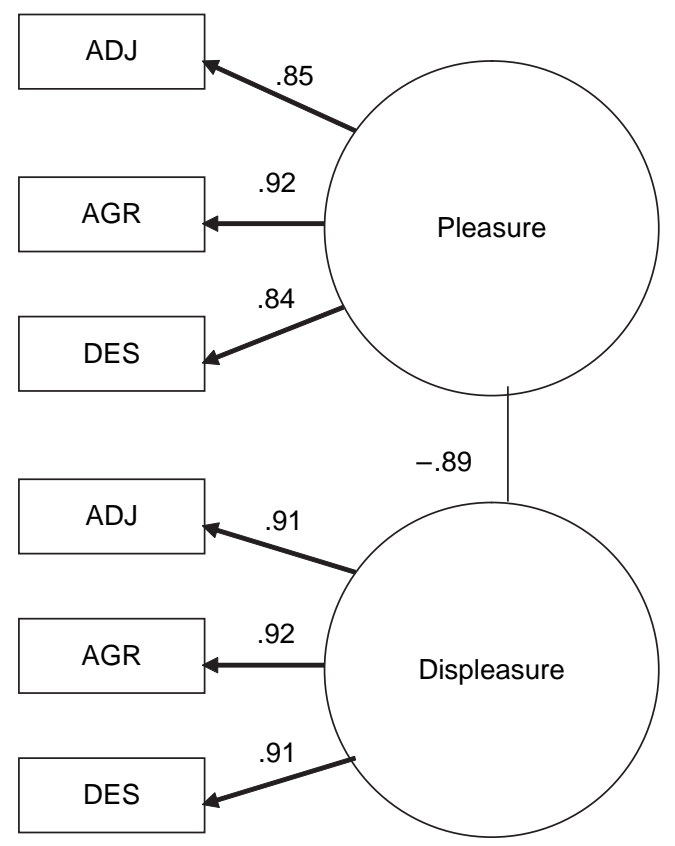

Figure 1. A confirmatory factor model for Pleasure and Displeasure constructs. Estimated coefficients significant at .001 are shown and were extracted from English Sample $1(N=217)$. Error terms and correlations between error terms with the same response format were estimated but are not shown.

\section{Measurement models using multiple response formats}

I next used confirmatory factor analysis to estimate the relation between Pleasure and Displeasure in a way that controlled for random and systematic measurement errors. Figure 1 displays the tested model, along with results from English Sample 1. In each model, I specified two latent constructs corresponding to Pleasure and Displeasure, each defined by three scales (manifest variables) each in a different response format. For all models, I estimated: (a) the factor loading between each manifest variable and its intended latent construct; (b) the error term associated with each manifest variable; (c) the correlation between the error terms associated with the same response format, and (d) the correlation between the two latent constructs. It is this last number $(\Phi$, a PHI coefficient) that estimates the correlation between hypothesised polar opposites.

Table 2 presents indices of fit and latent correlations between Pleasure and Displeasure. For all 24 models tested, the hypothesised model yielded an RMSSR between .01 and .03. Factor loadings for all models were 
TABLE 2

Results of confirmatory factor analysis: Pleasure-displeasure factor model using three different response formats

\begin{tabular}{|c|c|c|c|c|c|c|}
\hline \multirow[b]{2}{*}{ Language } & \multirow[b]{2}{*}{ Sample } & \multicolumn{4}{|c|}{ Indices of fit } & \multirow{2}{*}{$\begin{array}{l}\text { Latent correlation } \\
\text { between Pleasure and } \\
\text { Displeasure (SE) }\end{array}$} \\
\hline & & $\chi^{2}$ & $R M S S R$ & $A P G I$ & $C F I$ & \\
\hline \multirow[t]{3}{*}{ English 1} & Total & 19.96 & .02 & .92 & .99 & $-.89(.02)$ \\
\hline & Males & 7.60 & .03 & .94 & .99 & $-.87(.05)$ \\
\hline & Females & 20.57 & .02 & .88 & .98 & $-.91(.02)$ \\
\hline \multirow[t]{3}{*}{ English 2} & Total & 18.79 & .01 & .96 & 1.00 & $-.92(.01)$ \\
\hline & Males & 12.25 & .02 & .96 & 1.00 & $-.92(.02)$ \\
\hline & Females & 10.67 & .01 & .97 & 1.00 & $-.93(.01)$ \\
\hline \multirow[t]{3}{*}{ English 3} & Total & 15.52 & .01 & .96 & 1.00 & $-.93(.01)$ \\
\hline & Males & 15.14 & .01 & .91 & .99 & $-.93(.02)$ \\
\hline & Females & 13.20 & .01 & .95 & 1.00 & $-.94(.01)$ \\
\hline \multirow[t]{3}{*}{ Chinese 1} & Total & 9.52 & .01 & .99 & 1.00 & $-.88(.01)$ \\
\hline & Males & 8.34 & .01 & .97 & 1.00 & $-.84(.03)$ \\
\hline & Females & 6.16 & .01 & 1.00 & 1.00 & $-.90(.01)$ \\
\hline \multirow[t]{3}{*}{ Chinese 2} & Total & 9.56 & .01 & .98 & 1.00 & $-.88(.02)$ \\
\hline & Males & 3.15 & .01 & 1.00 & 1.00 & $-.85(.03)$ \\
\hline & Females & 10.07 & .02 & .97 & 1.00 & $-.91(.02)$ \\
\hline \multirow[t]{3}{*}{ Spanish } & Total & 9.01 & .01 & .98 & 1.00 & $-.89(.02)$ \\
\hline & Males & 3.88 & .01 & 1.00 & 1.00 & $-.83(.04)$ \\
\hline & Females & 16.09 & .02 & .90 & .99 & $-.94(.02)$ \\
\hline \multirow[t]{3}{*}{ Japanese } & Total & 19.67 & .02 & .96 & .99 & $-.77(.02)$ \\
\hline & Males & 12.32 & .01 & .96 & .99 & $-.71(.04)$ \\
\hline & Females & 9.98 & .02 & .97 & 1.00 & $-.83(.03)$ \\
\hline \multirow[t]{3}{*}{ Korean } & Total & 10.53 & .01 & .98 & 1.00 & $-.87(.02)$ \\
\hline & Males & 6.10 & .02 & .99 & 1.00 & $-.84(.03)$ \\
\hline & Females & 11.46 & .02 & .96 & 1.00 & $-.91(.02)$ \\
\hline
\end{tabular}

Note: $N$ for English $1=217$ (56 males and 161 females); $N$ for English $2=535$ (241 males and 294 females); $N$ for English $3=395$ (144 males and 251 females); $N$ for Chinese $1=487$ (164 males and 323 females); $N$ for Chinese $2=402$ ( 200 males and 202 females); $N$ for Spanish $=233$ (96 males and 137 females); $N$ for Japanese $=450$ (228 males and 222 females); $N$ for Korean $=365$ (176 males and 189 females).

$d f$ for each 2-factor model $=5$.

RMSSR $=$ Root Mean Square Standardised Residuals; APGI $=$ Adjusted Population Gamma Index; CFI = Comparative Fit Index; (SE) $=$ standard errors.

substantial and statistically significant, indicating that the manifest variables are reasonable indicators of their intended latent constructs. The last column of Table 2 shows the latent correlation between Pleasure and Displeasure in 
TABLE 3

Cross-gender comparisons of the relation between Pleasure and Displeasure

\begin{tabular}{lcccccccc}
\hline & \multicolumn{3}{c}{ Model 1 } & & \multicolumn{3}{c}{ Model 2 } \\
\cline { 2 - 3 } Language & $\chi^{2}$ & RMSSR & APGI & & $\chi^{2}$ & RMSSR & APGI & $\chi^{2}$ change \\
\hline English 1 & 33.52 & .05 & 0.95 & & 32.35 & .05 & 0.94 & 1.17 \\
English 2 & 34.00 & .11 & 0.97 & & 33.97 & .11 & 0.97 & 0.03 \\
English 3 & 30.81 & .01 & 0.97 & & 30.25 & .02 & 0.97 & 0.56 \\
Chinese 1 & 26.80 & .04 & 0.98 & & 21.69 & .04 & 0.99 & 5.11 \\
Chinese 2 & 20.33 & .03 & 1.00 & & 17.40 & .02 & 1.00 & 2.93 \\
Spanish & 36.30 & .08 & 0.95 & & 26.99 & .02 & 0.97 & $9.31^{*}$ \\
Japanese & 46.77 & .06 & 0.95 & & 39.10 & .03 & 0.96 & $7.67^{*}$ \\
Korean & 38.89 & .06 & 0.96 & & 35.20 & .04 & 0.96 & 3.69 \\
\hline
\end{tabular}

Note: Model $1(d f=17)$ refers to a multi-sample CFA with an equality constraint placed on the latent correlation between Pleasure and Displeasure in both gender samples. Model $2(d f=16)$ refers to a multi-sample CFA with the latent correlation between Pleasure and Displeasure separately estimated in each gender dataset.

RMSSR $=$ Root Mean Square Standardised Residuals; APGI =Adjusted Population Gamma Index.

$* p<.01$.

the total sample and in the gender subsamples; values ranged from -.71 to $-.94($ mean $=-.88){ }^{3}$

\section{Cross-gender comparisons}

To examine gender differences in each language sample, I created a twosample (males and females) confirmatory factor analysis with two latent factors (Pleasure and Displeasure), each indicated by three scales with different response formats. In Model 1, the parameter estimates for the factor loadings, and the inter-factor latent correlation were constrained to be equivalent in the two samples (males and females). In Model 2, the specifications were identical to those of Model 1 except that the inter-factor latent correlation was estimated separately for males and for females.

Results are presented in Table 3. I explain the results by looking at the data from the male and female subsamples of English Sample 1. Model 1 fits the data quite well: $\chi^{2}(17, N \mathrm{~s}=56,161)=33.52$, RMSSR $=.05, \Phi=$ -.90 ; Model 2 fits the data similarly well: $\chi^{2}(16, N \mathrm{~s}=56,161)=32.35$,

\footnotetext{
${ }^{3}$ I examined these measurement models using revised Pleasure and Displeasure scales in which semantic opposites were excluded. For instance, when happy was included in defining the Pleasure scale, unhappy was taken out from the Displeasure scale. Results were identical to those reported here and hence lend further support to the bipolarity of PA and NA.
} 
$\mathrm{RMSSR}=.05, \Phi \mathrm{s}=-.86$ for males and -.91 for females. The $\Delta \chi^{2}(1, N \mathrm{~s}=$ $56,161)=1.17$ and RMSSR did not change. Thus, estimating the latent correlation separately for males and females provided no improvement in fit. I therefore concluded that there was no significant gender difference in the latent correlation between Pleasure and Displeasure in English Sample 1. The same analysis sequence was repeated on the other samples. Significant gender differences were found in two samples: In both the Spanish and Japanese samples, female respondents showed a more bipolar relation between Pleasure and Displeasure than did male respondents, but, as shown in Table 2, the differences were tiny.

\section{Cross-cultural comparisons}

The results reported above justified combining the male and female data within each language sample. I further combined the three English samples into one and the two Chinese samples into one. I specified a five-sample (English, Chinese, Spanish, Japanese, and Korean languages) confirmatory factor analysis with two latent factors (Pleasure and Displeasure), each indicated by three scales with different response formats. To estimate the bipolarity coefficient in each language sample, I constrained the estimates of the factor loadings to be equivalent across all five datasets and estimated the latent correlation between Pleasure and Displeasure in each sample. The hypothesised model fit the data quite well: $\chi^{2}(49, N \mathrm{~s}=1147,889,233,450$, $365)=272.36$, $\operatorname{RMSSR}=.10$, APGI $=.95$. The differences in the bipolarity coefficients (ranging from -.80 to -.91 ) were tiny. In contrast to the dialectical model, I found that Aristotelian cultures did not differ significantly from Confucian ones.

\section{DISCUSSION}

To examine the cultural variation of the bipolarity thesis in momentary affect, I conducted a strict test of bipolarity in eight samples covering five languages. The bipolarity coefficients I obtained differ in magnitude and direction dramatically from those reported in previous research (e.g., Bagozzi et al., 1999; Schimmack et al., 2002). The coefficients for the total sample and for males and females separately were all negative and substantial in magnitude. Although these results do not allow me to declare that all pairs in the Pleasure and Displeasure scales were perfect polar opposites, the correlations do fit the pattern of expected polar opposites. Indeed, the latent correlations, ranging from -.80 to -.91 (mean $=-.87$ ), all fell within the predicted range of -.47 and -1.00 suggested by Russell and Carroll (1999). 
Consistent with Schimmack et al. (2002) but in contrast to Bagozzi et al. (1999), the present results suggested minimal gender differences in the PANA correlations. Bagozzi et al. found a complex pattern: In their English sample, females yielded more negative correlations than did males, but the opposite was the case in their Chinese and Korean samples in which female respondents yielded more positive correlations than did male respondents. In my eight samples, the results were simpler: In all cases (including the Aristotelian and Confucian samples), the correlations from female respondents were more negative (albeit not greatly so) than those from male respondents. Significant (but tiny) gender differences were found in the Spanish and Japanese samples.

Although researchers in the past pointed to the Confucian heritage or the naïve dialecticism of Eastern cultures, including China and Korea, to account for the shift towards more positive correlations in their samples, I found that shift, if anywhere, in the Japanese sample only.

Taken together, the present findings are consistent with the idea that momentary positive and negative affect per se are polar opposites (Scollon et al., 2005). Nevertheless, in remembering and hence reporting affective experience over an extended period of time, cultural and gender differences can be factors influencing the relation between PA and NA (Robinson \& Clore, 2002). I interpret Bagozzi et al.'s (1999) and Schimmack et al.'s (2002) results as consistent with the idea that in reporting the frequency of emotional experiences over a period of time (such as "in the past month" or even "right now") using the two abstractions of PA and NA, respondents from Eastern (or at least Japanese) cultures tend to recall opposite feelings, to believe them reconcilable, and therefore to report them at the same time (Peng \& Nisbett, 1999). In reporting emotional experiences for a recent and isolated "slice" of time, however, representations of positive and negative affect are indeed polar opposites.

Manuscript received 1 December 2004 Revised manuscript received 24 May 2006 Manuscript accepted 24 May 2006

\section{REFERENCES}

Bagozzi, R. P., Wong, N., \& Yi, Y. (1999). The role of culture and gender in the relationship between positive and negative affect. Cognition and Emotion, 13, 641-672.

Bradburn, N. M. (1969). The structure of psychological well-being. Chicago: Aldine.

Brislin, R. W. (1970). Back-translation for cross-cultural research. Journal of Cross-Cultural Psychology, 1, 185-216.

Brody, L. R., \& Hall, J. A. (1993). Gender and emotion. In M. Lewis \& J. M. Haviland (Eds.), Handbook of emotions (pp. 447-460). New York: Guilford. 
Cacioppo, J. T., \& Bernson, G. G. (1994). Relationship between attitudes and evaluative space: A critical review, with emphasis on the separability of positive and negative substrates. Psychological Bulletin, 115, 401-423.

Crystal, D. (1997). The Cambridge encyclopedia of language (2nd ed.). New York: Cambridge University Press.

Diener, E., \& Emmons, R. A. (1984). The independence of positive and negative affect. Journal of Personality and Social Psychology, 47, 1105-1117.

Diener, E., Smith, H., \& Fujita, F. (1995). The personality structure of affect. Journal of Personality and Social Psychology, 69, 130-141.

Feldman Barrett, L., \& Russell, J. A. (1998). Independence and bipolarity in the structure of current affect. Journal of Personality and Social Psychology, 74, 967-984.

Fredrickson, B. L. (2001). The role of positive emotions in positive psychology: The broaden and build theory of positive emotions. American Psychologist: Special Issue, 56, 218-226.

Green, D. P., Goldman, S. L., \& Salovey, P. (1993). Measurement error masks bipolarity in affect ratings. Journal of Personality and Social Psychology, 64, 1029-1041.

Kitayama, S., Markus, H. R., \& Kurokawa, M. (2000). Culture, emotion, and well-being: Good feelings in Japan and the United States. Cognition and Emotion, 14, 93-124.

Larsen, J. T., McGraw, A. P., \& Cacioppo, J. T. (2001). Can people feel happy and sad at the same time? Journal of Personality and Social Psychology, 81, 684-696.

Larsen, R. J., \& Diener, E. (1992). Promises and problems with the circumplex model of emotion. In M. S. Clark (Ed.), Review of Personality and Social Psychology: Emotion (Vol. 13, pp. 25-59). Newbury Park, CA: Sage.

Lutz, C. A. (1996). Engendered emotion: Gender, power, and the rhetoric of emotional control in American discourse. In R. Harré \& W. G. Parrott (Eds.), The emotions (pp. 151-170). London: Sage.

Markus, H. R., \& Kitayama, S. (1991). Culture and the self: Implications for cognition, emotion, and motivation. Psychological Review, 98, 224-253.

Peng, K., \& Nisbett, R. E. (1999). Culture, dialectics, and reasoning about contradictions. American Psychologist, 54, 741-754.

Robinson, M. D., \& Clore, G. L. (2002). Episodic and semantic knowledge in emotional selfreport: Evidence for two judgment processes. Journal of Personality and Social Psychology, 83, 198-215.

Russell, J. A. (1980). A circumplex model of affect. Journal of Personality and Social Psychology, $39,1161-1178$.

Russell, J. A., \& Carroll, J. M. (1999). On the bipolarity of positive and negative affect. Psychological Bulletin, 125, 3-30.

Schimmack, U., Oishi, S., \& Diener, E. (2002). Cultural influences on the relation between pleasant emotions and unpleasant emotions: Asian dialectic philosophies or individualismcollectivism? Cognition and Emotion, 16, 705-719.

Scollon, C. N., Diener, E., Oishi, S., \& Biswas-Diener, R. (2005). An experience sampling and cross-cultural investigation of the relation between pleasant and unpleasant affect. Cognition and Emotion, 19, 27-52.

Spencer-Rodgers, J., Peng, K., Wang, L., \& Hou, Y. (2004). Dialectical self-esteem and EastWest differences in psychological well-being. Personality and Social Psychology Bulletin, 30, $1416-1432$.

Spiegel, D. (1998). Getting there is half the fun: Relating happiness to health. Psychological Inquiry, 9, 66-68.

Thayer, R. E. (1996). The origin of everyday moods: Managing energy, tension, and stress. New York: Oxford University Press.

Watson, D., \& Tellegen, A. (1985). Toward a consensual structure of mood. Psychological Bulletin, 98, 219-235. 
Yik, M., \& Russell, J. A. (2001). Predicting the Big Two of affect from the Big Five of personality. Journal of Research in Personality, 35, 247-277.

Yik, M., \& Russell, J. A. (2003). Chinese affect circumplex: I. Structure of recalled momentary affect. Asian Journal of Social Psychology, 6, 185-200.

Yik, M., Russell, J. A., \& Ahn, C.-K. (2003a). Affect among Koreans: New scales and their structure. Korean Journal of Psychology, 22, 115-136.

Yik, M., Russell, J. A., Ahn, C.-K., Fernández Dols, J. M., \& Suzuki, N. (2002). Relating the Five-Factor Model of personality to a circumplex model of affect: A five language study. In R. R. McCrae \& J. Allik (Eds.), The Five-Factor Model of personality across cultures (pp. 79 104). New York: Kluwer Academic/Plenum Publishers.

Yik, M., Russell, J. A., \& Feldman Barrett, L. (1999). Structure of self-reported current affect: Integration and beyond. Journal of Personality and Social Psychology, 77, 600-619.

Yik, M., Russell, J. A., Oceja, L. V., \& Fernández Dols, J. M. (2000). Momentary affect in Spanish: Scales, structure, and relation to personality. European Journal of Psychological Assessment, 16, 160-176.

Yik, M., Russell, J. A., \& Steiger, J. H. (2007). A 12-point circumplex model of core affect. Manuscript submitted for publication.

Yik, M., Russell, J. A., \& Suzuki, N. (2003b). Relating momentary affect to the five factor model of personality: A Japanese case. Japanese Psychological Research, 45, 80-93.

Zautra, A. J., Potter, P. T., \& Reich, J. W. (1997). The independence of affects is contextdependent: An integrative model of the relationship between positive and negative affect. In M. P. Lawton \& K. W. Schaie (Eds.), Annual Review of Gerontology and Geriatrics (pp. 75102). NewYork: Springer. 